\title{
The Genetic Underpinnings of Body Fat Distribution
}

Sara L. Pulit ${ }^{1,2,6 *}$, Samantha Laber ${ }^{1,3,4 *}$, Craig A. Glastonbury ${ }^{1,5}$, Cecilia M. Lindgren ${ }^{1,5,6}$

\section{Affiliations}

1. Big Data Institute, Li Ka Shing Centre for Health Information and Discovery, University of Oxford, Oxford, U.K.

2. Department of Genetics, University Medical Center Utrecht, Utrecht, The Netherlands.

3. MRC Harwell Institute, Mammalian Genetics Unit, Harwell, Oxford, U.K.

4. Department of Physiology, Anatomy and Genetics, University of Oxford, Oxford, U.K.

5. Wellcome Trust Centre for Human Genetics, Nuffield Department of Medicine, University of Oxford, Oxford, U.K.

6. Program in Medical and Population Genetics, Broad Institute, Cambridge, Massachusetts, USA

\section{Corresponding Author}

Prof. Cecilia M. Lindgren

The Big Data Institute, Li Ka Shing Centre for Health Information and Discovery

University of Oxford

Roosevelt Drive

Oxford

OX3 $7 \mathrm{BN}$

United Kingdom

celi@well.ox.ac.uk 


\begin{abstract}
Introduction

Obesity, defined as a body mass index (BMI) > 30 kilograms/meter ${ }^{2}$, has reached epidemic proportions: people who are overweight $\left(\mathrm{BMI}>25 \mathrm{~kg} / \mathrm{m}^{2}\right)$ or obese now comprise more than $25 \%$ of the world's population. Obese individuals have a higher risk of comorbidity development including type 2 diabetes, cardiovascular disease, cancer, and fertility complications.
\end{abstract}

\title{
Areas covered
}

The study of monogenic and syndromic forms of obesity have revealed a small number of genes key to metabolic perturbations. Further, obesity and body shape in the general population are highly heritable phenotypes. Study of obesity at the population level, through genome-wide association studies of BMI and waist-to-hip ratio (WHR), have revealed $>150$ genomic loci that associate with these traits, and highlight the role of adipose tissue and the central nervous system in obesity related traits. Studies in animal models and cell lines have helped further elucidate the potential biological mechanisms underlying obesity. In particular, these studies implicate adipogenesis and expansion of adipose tissue as key biological pathways in obesity and weight gain.

\section{Expert commentary}

Further work, including a focus on integrating genetic and additional genomic data types, as well as modeling obesity-like features in vitro, will be crucial in translating genome-wide association signals to the causal mechanisms driving disease. 


\section{Key issues}

- Obesity is now a global epidemic, affecting more than $25 \%$ of the world's population. Obesity associates with a number of adverse health outcomes including type 2 diabetes, hypertension, stroke, and several types of cancer.

- The study of obesity has been conducted through measurement of a number of fat distribution phenotypes including body mass index (BMI), waist-to-hip ratio (WHR), WHR adjusted for BMI (WHRadjBMI), and waist circumference. Additionally, imaging approaches such as magnetic resonance imaging allow for more refined phenotypic measurements, such as the volume of adipose tissue collected in particular locations in the body (called 'depots.')

- A number of genes have been implicated in monogenic and syndromic forms of obesity. Additionally, BMI, WHRadjBMI, and other fat distribution measurements have been demonstrated to be highly heritable (heritability of BMI is $~ 80 \%$; heritability of WHRadjBMI is $~ 30-45 \%)$. Genome-wide association studies of these phenotypes in the general population have revealed 97 common variant loci associated to BMI and an additional 49 loci associated to WHRadjBMI.

- Animal models and cell models have yielded additional insight into the biology of obesity and specifically to the disease-conferring effects of subcutaneous and visceral adipose tissue. Further, these models have shown how adipose tissue expansion changes in mice on a high-fat diet and how, in some instances, the mechanisms of adipose tissue expansion are sexually dimorphic.

- While genome-wide association studies and animal models have implicated a number of common variants associated to obesity-related traits and provided insights into the mechanisms of adipose tissue expansion, further work is necessary to unravel the key genes and pathways that regulate adipose tissue accumulation and deposits.

- The discovery of the regulatory mechanism at the FTO locus, the first locus demonstrated to be associated to BMI in the general population, demonstrates how integration of genetic and genomic data - including genetic variation, gene expression, chromatin folding, and CRISPR/Cas experiments - will be crucial to uncovering the biological mechanisms of obesity and identifying potential therapeutic targets. 


\section{Obesity as a global pandemic}

Worldwide, obesity has reached pandemic proportions. The number of individuals who are either overweight (body mass index, BMI > $25 \mathrm{~kg} / \mathrm{m}^{2}$, as defined by the World Health Organization) or obese $\left(\mathrm{BMI}>30 \mathrm{~kg} / \mathrm{m}^{2}\right)$ has increased to over 1.9 billion, more than $25 \%$ of the world's population $[1,2]$. Though the epidemic has been a primary public health concern in developed nations, equally concerning is the impact of obesity in the developing world, where rates of obesity have also been increasing $[1,3]$. While obesity rates still remain lower in developing nations than rates in developed countries, nearly two-thirds of the world's obese population reside in developing countries [3].

Overweight and obesity associates with overall morbidity and mortality. Obese individuals are at higher risk of a number of diseases including type 2 diabetes (T2D), hypertension, stroke, cardiovascular disease, and several types of cancer [3,4]. Obesity also correlates with fertility and pregnancy complications (like gestational diabetes), which can have both short and longer-term impact on the health of both mother and offspring [5]. While some studies have suggested the notion of 'metabolically healthy obesity' -- observed in individuals who are obese but experience no adverse health outcomes -- recent large-scale meta-analyses suggest that there is no genetic evidence for metabolically healthy obesity and that all obesity is, in fact, disease risk increasing [6]. Given the long-term health implications for individuals who are either overweight or obese, the rising prevalence of obesity in children worldwide poses a profound threat to the affected population and to overall public health [7], now and in the future.

Here, we review critical aspects of the study of obesity, including the many ways in which obesity can be measured, and the known genetic contributions to obesity both in monogenic disease as well as in obesity at the population level (i.e., as a polygenic trait). We additionally discuss the known molecular mechanisms driving adipose tissue expansion. Finally, we propose ways in which current understanding of obesity, combined with novel technologies, increasing data, and method development can be used to further understand obesity biology, inform novel research lines, and elucidate potential drug targets. 


\section{Measuring obesity in epidemiology and biology}

To understand and analyze the health implications of being overweight or obese, and to unravel where fat is deposited in humans and the mechanisms that regulate these deposits, researchers must select from a number of phenotypic measurements from which to study (Figure 1). There are several commonly-used anthropometric traits that are frequently used in the study of obesity and searching for genetic variation that influence adipose-related measures; BMI [8], waist-to-hip ratio (WHR [9]) and waist circumference (WC [9-11]), as they are generally readily measurable, and can be easily ascertained in hundreds of thousands of individuals (Figure 1).

While anthropometric measures are informative, non-invasive to collect, and globally applicable, they provide an incomplete view of the distribution of adipose tissue in humans. Consequently, more refined phenotypic measures have emerged across the field of obesity research, as scientists grapple with establishing more detailed obesity-related measures that may yield additional biological insights into obesity and metabolic traits. Specifically, researchers have sought to measure the amount of adipose tissue residing in particular locations (or depots) throughout the body. Imaging methods such as magnetic resonance imaging (MRI) or computed tomography (CT) scans allow for the measurement of visceral and subcutaneous adipose tissue (VAT and SAT, respectively), as well as adipose tissue residing around particular organs, such as the heart (called pericardial adipose tissue, or PAT) (Figure 1). Further still, these phenotypic measures can be adjusted for BMI (e.g., WHRadjBMI or VATadjBMI) to ascertain the phenotypic measures independent of the influence of overall BMI. While this may be useful in accounting for the effects of BMI, this method also introduces the potential for collider bias [12], a phenomenon in which adjustment for causally related covariates can induce replicable, yet biologically spurious, associations [12,13]. Notably, none of the individually reported genetic variants for WHRadjBMI show evidence of collider bias, but the potential for collider bias implies the necessity for caution when interpreting results for adjusted phenotype associations.

Lastly, behavioural phenotypes, such as caloric intake, feeding habits and patterns, and physical activity, also provide a means of measuring obesity, obesity-related outcomes, and metabolism. These phenotypes, unlike the anthropometric measures described above, typically depend on survey or interview data. Consequently, these measures are likely more difficult to study, as their 
accuracy depends upon, for example, memory of and accuracy regarding eating behaviour and physical activity; humans tend to inaccurately report both [14].

\section{Underlying biology in obesity and adipose-related traits: genetic variation}

While scientists are capable of measuring a number of physical attributes as a means of approximating overall fat distribution or the amount of adipose tissue residing in a specific depot, additional study is necessary to determine whether these measurements are modulated by genetic factors, environmental factors, or both. Establishing a role for genetics in these phenotypes is a critical first step in understanding the biological drivers of obesity and related disease outcomes.

\section{i. Obesity as a feature of monogenic and syndromic disease}

A rare number of individuals are affected by typically early onset, syndromal forms of disease, hallmarked, at least in part, by extreme obesity. These diseases are attributable essentially entirely to genetic factors: the disease can segregate in families and be pinpointed to a particular mutation or sets of mutations residing in specific genes. Various mutations in the POMC, leptin $(L E P)$, leptin receptor ( $L E P R)$, and other genes responsible for hormone processing have been implicated in monogenic forms of obesity [15]. For example, some individuals carry frameshift mutations that make them deficient for either LEP or LEPR; these individuals are of normal weight at birth, but become rapidly obese in the months following [16].

Additionally, mutations in the melanocortin 4 receptor $(M C 4 R)$ gene on chromosome 18 have been found to segregate in both an autosomal dominant and autosomal recessive manner in individuals with monogenic obesity $[17,18]$. Genetic mutations in $M C 4 R$ were among the first implicated in monogenic obesity-related syndromes. These mutations can be found in a number of global populations at varying frequencies. A study of 2,000 severely-obese individuals found that pathogenic mutations were carried in $\sim 5 \%$ of the individuals $[16,19]$. Additional studies in European-ancestry populations estimate that $M C 4 R$ mutations can be found in $1.0-2.5 \%$ of obese individuals, indicating that mutations in $M C 4 R$ are the most common form of monogenic obesity [16].

Other rare diseases, including Prader-Willi Syndrome (affecting 1 in 25,00o live births) [15], Cohen syndrome (affecting < 500 people worldwide) [9], and Bardet-Biedl Syndrome (affecting 
$<1$ in 13,000 individuals) [20], can all include onset of obesity as one of their clinical features. Intriguingly, not all individuals with Bardet-Biedl syndrome become obese, suggesting incomplete penetrance of disease-causing mutations, the interplay with other genetic or environmental factors, or a combination of these [15].

Though monogenic and syndromic forms of obesity range in their clinical presentation and can additionally include developmental abnormalities (such as polydactyly), mental retardation, or dysmorphic physical features, the underlying genetic causes share a common thread: the mutations fall into the leptin-melanocortin pathway [21]. The pathway, governed by the central nervous system, helps regulate feeding and fasting, and highlights a potentially important role for the nervous system in monogenic or syndromic forms of obesity [21]. In addition, studies of monogenic and syndromic forms of obesity have revealed key genes that appear to play a role in body fat distribution in the general population.

\section{ii. Heritability of obesity and related traits in the general population}

While monogenic and syndromic forms of obesity show clear inheritance patterns across families, heritability estimates indicate that BMI and other body fat distribution measures have a (strong) heritable component. Thus, these features in the general population are influenced in part by genetic factors. Heritability estimates can vary widely, as they are influenced by a number of factors including the samples and sample size selected for study, methodological approaches for measuring heritability, and confounders [22,23]. Despite the potential imprecision of such estimates, heritability estimates for height, weight, and BMI tend to converge around particular estimates. Height is highly heritable, with an estimated 80\% of variation in the trait explained by genetic factors [24-27]. The heritability of weight is estimated to be $\sim 70 \%[24,28]$. BMI is also highly heritable; heritability estimates vary somewhat more broadly than for height, and heritability is estimated to be between 40 and $80 \%$ [21,24,29,30]. Notably, heritability estimates for BMI vary with the relatedness of the samples tested and tend to be higher in twins compared to estimates derived from families or from genotyping data used for genome-wide association analyses [21,29].

Phenotypes meant to better capture fat distribution have also been demonstrated to be heritable, though more modestly so. WC and WHR are estimated to be $30-45 \%$ heritable, even after adjusting for BMI $[9,21,28]$. Additionally, the amount of VAT and SAT are also heritable 
features (h2 $=36 \%$ and 57\%, respectively) [10]. Importantly, heritability estimates in WC adjusted for BMI (WCadjBMI), WHR and WHRadjBMI differ in males and females; in contrast, heritability estimates in BMI, height, WC and weight are stable across the sexes [28]. The biological explanation for the sexually dimorphic signals has not yet been fully elucidated. However, these sex differences point to potentially distinct biological mechanisms in males and females (e.g., the potential importance of hormones, or potential selection for genes that confer an 'optimal' WHR in females) in some body distribution traits, while other traits are more likely to share mechanisms across the sexes.

\section{iii. Obesity as a common disease}

Having established that body fat distribution traits are heritable, we are able to search for the biological mechanisms or genetic factors that influence these traits. To date, the most efficient means of identifying common genetic variation that influence common traits and diseases is genome-wide association studies (GWAS) [31]. The last decade of genetic research in common disease has been characterized in large part by GWAS and downstream meta-analysis of GWAS data from many cohorts. Studies have now genotyped and tested millions of common (minor allele frequency > 1\%) single-nucleotide polymorphisms (SNPs) in hundreds of thousands of samples. These efforts have revealed thousands of mostly modest-effect (odds ratios ranging from 1.05-1.50 [32]) variants associated with hundreds of traits and diseases [33].

GWAS of BMI and other body fat distribution traits began at the start of the GWAS era, and the discovery of a common variant in the FTO gene [34] as associated to BMI was one of the first robust genetic discoveries of this era. The top implicated SNP (rs9939609) resides in an intron of FTO, and confers a rather large effect on BMI (also likely explaining why it was one of the first-discovered SNPs of the GWAS era): each additional allele of the SNP is estimated to increase BMI by $\sim 0.4 \mathrm{~kg} / \mathrm{m}^{2}$. The study initially implicating FTO, which performed a GWAS in > 20,000 individuals of European ancestry, additionally found that carriers for the risk allele of rs9939609 were, on average, $\sim 3 \mathrm{~kg}$ heavier and had higher risk of obesity compared to individuals who were homozygous for the non-risk allele [34].

A subsequent GWAS, performed one year later and now interrogating > 60,000 individuals of European descent, identified common markers in the genomic region of the $M C 4 R$ gene that also associated with BMI [35]. The finding near $M C 4 R$ highlighted the intriguing possibility 
that, while rare mutations in $M C 4 R$ undoubtedly confer risk for monogenic obesity, common variants in the region are also influencing BMI at the population level and, consequently, regulation or altered expression of $M C 4 R$ is potentially important for both rare and common forms of obesity [35]. Following this GWAS, a third one, performed in 2009 in nearly 100,000 individuals, confirmed the associations discovered in and near FTO and MC4R and discovered six novel loci [36]. The effort additionally demonstrated, through construction of a genetic risk score, that individuals with higher BMI do indeed carry more risk-increasing alleles than individuals with lower BMI. This genetic risk score was insufficient, however, to predict an individual's BMI from common genetic variants alone, indicating the challenge of predicting individuals at higher risk of obesity from genetic data alone.

Since these initial genome-wide association studies, analysis of BMI and other body shape or fat distribution traits have continued apace. The latest GWAS of BMI and WHRadjBMI, both completed in 2015, studied > 330,000 individuals and implicated 97 loci and 49 loci, respectively, as associated to the traits. Additionally, GWAS have been performed using body fat traits such as SAT and VAT, identifying novel associated loci as well as loci already implicated in studies of BMI and WHRadjBMI [10]. Recent GWAS are also beginning to leverage the phenotyping made available through imaging approaches such as MRI, and are using these image-based phenotypes (e.g., SAT measured through MRI) as a means of discovering newly-associated loci [37].

Though these GWAS interrogate traits that all, in some way or another, reflect fat distribution and body shape, the discoveries and biological inferences gleaned from these discoveries often vary. In fact, increasingly larger GWAS in adipose-related traits have revealed quite distinct genetic signatures underlying each trait, suggesting that different phenotypic measures capture different biological mechanisms that underpin the highly complex phenotypes that are obesity and adipose-related features. Perhaps the most striking example of these differences are the insights drawn from the most recent GWAS in BMI and WHRadjBMI [8,9]. Both GWAS were performed in a (mostly-overlapping) set of > 330,000 samples (primarily of European ancestry) collected by the GIANT consortium. The analysis of BMI revealed a total of 97 loci associated to BMI; all of these loci, with the exception of 3 , were directionally consistent in effect and association across the sexes. Further, DEPICT [38] analyses of the implicated loci, used to elucidate potential pathways underlying the various associated loci, revealed a role for the 
central nervous system in the biology of BMI [8]. The authors inferred from these findings that key neurological pathways govern obesity biology, for example through the regulation of feeding and fasting signals.

In contrast, GWAS of WHRadjBMI revealed a total of 49 associated loci, a large proportion of which were sexually dimorphic. Specifically, an impressive 20 of the 49 loci showed a stronger association signal in sex-specific analysis. Perhaps even more striking, 19 of the 20 sexually-dimorphic loci were strongly associated in females only [9], all with directions of effect increasing WHRadjBMI. The finding confirmed a prominent role for sexually-dimorphic loci, a feature of WHRadjBMI genetic architecture that had been hinted at in previous GWAS of WHRadjBMI and WCadjBMI [11]. Further, a suite of pathway analysis approaches were used to discover a role for mesenchyme (i.e., connective) tissues, including adipose tissue, in the biological mechanisms underpinning WHRadjBMI [9]. These tissues have also been implicated in the study of type 2 diabetes [39] and lipids [40,41], traits for which many of the WHRadjBMI-implicated loci overlap [9]. An additional GWAS in SAT, VAT and a number of other specific depots has also confirmed the key role for adipocytes and adipogenesis in these fat distribution phenotypes [37]. Thus, while recent analysis of BMI has revealed a role for both adipose tissue and the central nervous system in overall adiposity (consistent with monogenic forms of obesity), WHRadjBMI captures signals enriched for biological mechanisms originating in adipose tissue.

A recent GWAS of ectopic fat depots in $\sim 18,300$ individuals further highlights the disparate biology captured by various adipose-related phenotypes. The analysis focused on a number of depots including visceral and subcutaneous adipose tissue (VAT and SAT, respectively), as well as PAT, the ratio of VAT and SAT, and VAT and SAT as measured through MRI [37]. To directly interrogate the genetic overlap or distinctness of ectopic fat depot genetics and the genetics of BMI and WHRadjBMI, the group compared the 27 nominally-significant $(\mathrm{p}<0.05)$ loci associated to SAT with the loci discovered in BMI and WHRadjBMI. All 27 SNPs were directionally consistent with the BMI findings, despite having p-values well below genome-wide significance [37]. None of the other traits the authors investigated, however, showed such an overlap with either the BMI or the WHRadjBMI loci. Thus, while SAT and BMI appear to be genetically concordant, the other traits analyzed in this effort appear to be capturing additional, separate features of adipose biology [37]. Notable here, the analysis of ectopic fat depots had a substantially smaller sample size than that of BMI and WHRadjBMI, and analyses in 
comparatively-powered samples (i.e., >300,000 individuals) may reveal more commonality across the phenotypes.

More recently, efforts to understand the biology of obesity have expanded beyond GWAS to analyze methylation and its potential role in obesity. An epigenome-wide analysis of 5,387 people discovered $278 \mathrm{CpG}$ sites in 207 loci that associated with BMI [42]. Investigation of these signals across three metabolically-relevant tissues - blood, liver, and adipose - revealed correlation of the signals across the tissues. Additionally, application of Mendelian Randomization and a genetic risk score built from BMI-associated SNPs revealed that a subset of the loci were causal to increased BMI (rather than a consequence of it). Finally, the authors used a methylation risk score to reveal that epigenome-wide data could be used in a clinical setting: the score was strongly predictive of individuals at risk of individuals with incident T2D, contributed predictive qualities beyond that of standard clinical features that are typically considered, and in particular could identify individuals who are overweight or obese who are at particularly high risk of T2D [42].

While the many GWAS and the epigenome-wide study highlighted here have studied an array of body fat phenotypes, their findings yield a number of key, distinct insights. In particular, the loci implicated highlight a complex genetic interplay of some shared genetic signatures across BMI, WHRadjBMI, and other metabolic traits [8,9,25,39,41,43,44]. Beyond this interplay, however, there are also unique genetic signatures underpinning the traits, implicating distinct roles of the central nervous system and adipogenesis. The sexually dimorphic signals in WHRadjBMI also indicate that these roles may be regulated uniquely depending on the sex of the individual. Importantly, the more recent analysis of new data types (the epigenome-wide study was published in 2017) reveals the importance of studying additional biological data along with SNP array data; this new data can reveal genetic signatures, key pathways, and important clinical implications [42] that may be missed by array-focused analyses such as GWAS.

\section{Adipose tissue development and expansion in obesity}

While genome-wide association studies have revealed a host of associations in obesity and obesity-related traits, these studies mark, in essence, only the beginning of our understanding of the biology of obesity. GWAS findings have made clear that adipose tissue and mesenchymal stem cells are two of several key effectors of genetic risk loci for obesity trait associations and 
related traits (such as T2D), and in particular for body fat distribution signals $[9,45,46]$. Disentangling the full biological meaning of an association discovered through GWAS, however, remains a primary challenge in the study of complex traits and common disease. Key questions, including the target genes of implicated SNPs, the relevant tissues, and the important developmental stages of cells and tissues, all remain open. Additionally, the sexually-dimorphic signatures discovered in body fat features but not in overall BMI remain to be understood. To begin fully disentangling the biology of obesity, one key feature demands immediate understanding: the mechanisms that underlie white adipose tissue (WAT) growth in vivo. Specifically, we need to understand and study differences in WAT origin, depot, biological sex, development and response to stimuli.

In humans and rodents, white adipose tissue can be classified into two main types: subcutaneous (SAT) and visceral or intra-abdominal (VAT) fat. SAT can further be subclassified into deep and superficial adipose tissue, relating to its anatomical location (Figure 1). Furthermore, surplus fat can also deposit ectopically, mainly within the liver and skeletal muscle. Whether there are progenitors in muscle that differentiate into intra-muscular adipocytes or whether muscle cells take up lipid is currently unclear.

\section{i. White adipose tissue (WAT) distribution}

The distribution of WAT into SAT and VAT depots contributes differentially to cardiometabolic disease risk. Increasing visceral fat mass is associated with adverse metabolic outcomes across ancestries [47-52] whereas excess subcutaneous fat is believed to have no effect or potentially protective properties for metabolic disease [53-55]. For example, elevated SAT mass has been linked to improved blood pressure, plasma lipid profiles, insulin sensitivity, and atherosclerosis [54,56-6o]. These findings indicate that the mechanisms governing depot-specific expansion of WAT are directly relevant to metabolic health and disease.

Differences between SAT and VAT have historically been partly attributed to their anatomical location, which has implications in venous drainage [61]. However, over the past years, it has become clear that SAT and VAT display an array of differences including gene expression of fundamental development and patterning genes [62-67], developmental lineage [68-70], metabolic characteristics [55,67], levels of adipokine secretion, lipolysis rate, and tendency to develop inflammation [61]. Additionally, a body of evidence reports a differential adipose 
distribution between males and females. Males tend to accumulate fat in visceral depots around the abdomen, whereas premenopausal women preferentially accumulate SAT around the hips and gluteal regions [71]. In humans, this differential fat distribution pattern has been described as "apple-shaped" (centrally-deposited fat) and "pear-shaped" (hip- and gluteal-deposited fat) [72]. In women that are post-menopausal, WAT distribution mimics the visceral obesity pattern often observed in males, suggesting that sex hormones drive the balance between SAT and WAT expansion [71]. However, the cellular and molecular processes underlying the depot- and sex-specific expansion of WAT in vivo are currently not well understood.

\section{ii. Differences in WAT plasticity across depots in obesity}

WAT can expand via two distinct mechanisms: increased lipid filling in existing adipocytes (hypertrophy) or increased adipocyte number (hyperplasia). Mature adipocytes are post-mitotic; therefore, hyperplastic growth relies on de novo adipogenesis of adipocyte precursors that reside around the vasculature within the fat tissue [73]. Adipocyte hypertrophy, but not total adipose tissue mass, is typically linked to elevated risk of developing T2D and positively correlated with glucose intolerance and hyperinsulinaemia [74]. Furthermore, large, lipid-laden adipocytes are more likely to trigger an inflammatory response [75] and obese individuals with T2D typically have larger adipocytes in both SAT and WAT [76]. From carbon-14 birth-dating experiments, we know that the adipocyte turnover in SAT is $10 \%$ per year. In obese individuals, approximately twice as many adipocytes are turning over compared to lean individuals (that is, the half-life of an adipocyte is half as long), suggesting that obese individuals have double the amount of adipocytes [77]. Interestingly, adipocyte number remains higher in obese individuals, even after severe weight loss, suggesting that obesity-induced adipogenesis in adulthood has lifelong effects on WAT mass and homeostasis.

Additional work suggests that expansion of adipose tissue goes in hand with growth of additional blood vessels (called angiogenesis), in order to maintain supply to the increased demand resulting from the increase in adipose tissue. The angiogenic response is believed to be mediated via macrophages that are recruited following local hypoxia within the growing adipose tissue. In fact, hypoxia is one of the biggests microenvironmental factors of angiogenesis in WAT [78]. In contrast, mice that lack proinflammatory cytokine TNFa in adipose tissue present with reduced angiogenesis and adipose tissue expansion following a diet high in fat [79], suggesting that inflammation and angiogenesis play a critical role 
in WAT expansion. This observation is intriguing, given that, in addition to adipogenesis, angiogenesis was one of the pathways implicated through downstream analysis of body fat distribution loci discovered through GWAS of WHRadjBMI.

In humans and mice, the expansion of VAT in males occurs mainly through adipocyte hyperplasia [80-82]. In contrast, male SAT does not undergo hyperplastic growth in response to obesogenic stimuli such as a high-fat diet [80,82] (Figure 2). Using the 'AdipoChaser' mouse model, Wang et al. [82] reported that within the first months of a high-fat dietary challenge, the main contributor to WAT expandability is through adipose tissue hypertrophy. An extended exposure to a diet high in fat will then lead to hyperplasia/adipogenesis in VAT, but not SAT (Figure 2). Consistent with this finding, Jeffery et al. [80] reported that after 8 weeks of high-fat diet feeding, adipogenesis occurred exclusively in VAT. Importantly, in young animals, a high-fat diet leads to increased adipocyte size in both SAT and VAT. However, in adult mice, only VAT undergoes hyperplasia. This finding suggests that an age-related failure of SAT progenitor plasticity could underlie this phenomenon and the consequent metabolic disease risk [83].

The processes underlying depot-specific adipocyte progenitor activation are unclear. However, it is widely observed that adipocyte progenitors from different depots have differential differentiation capacity. Adipocyte precursors isolated from SAT differentiate more readily than adipocyte precursors from VAT, suggesting that hypertrophy preferentially happens in VAT as a result of the limited capacity of adipocyte progenitor recruitment in VAT and resistance to adipogenic stimulants. In contrast, SAT expands through hyperplasia due to higher pre-adipocyte number and/or adipogenic capacity. Additional work suggests that the abundance of precursors in SAT is a determinant of SAT expandability, while it correlates negatively with VAT accumulation in humans [84]. Thus, the ability to expand the SAT via de novo adipogenesis in adult life appears to protect from adverse metabolic outcomes. However, how an individual's genetic background contributes to one's ability to respond to environmental triggers is currently largely unknown. GWAS findings to date suggest that body fat distribution, i.e., the particular depots in which we store fat, has a strong genetic underpinning (discussed above).

In addition to the way we expand white adipose tissue and where in the body it gets distributed, genetics also influence its thermogenic activity. White adipose tissue is plastic and can be triggered to become more brown-like, as apparent via smaller, multi-locular adipocytes with 
increased amount of mitochondria and the capacity to produce heat. There is currently evidence for both, trans-differentiation of white into brown-like adipocytes or differentiation of inducible brown adipocyte precursors residing within white depots (reviewed in [85]). Adipocyte browning and consequently increased thermogenic capacity within white adipose tissue is associated with reduced metabolic disease and obesity and has therefore gained attention as being of therapeutic potential (reviewed in [86]).

\section{iii. Differences in WAT expansion between sexes}

Sexual dimorphism in obesogenic WAT growth [9] has direct implications in obesity-associated pathologies. The preferential accumulation of VAT in males has adverse metabolic health outcomes, whereas accumulation of SAT in females may be protective. High-fat diet feeding in mice results in sex-specific patterns of adipogenesis and that this response contributes to the differential fat distribution observed across the sexes[87] (Figure 2). Specifically, a high-fat diet induced adipogenesis in male VAT, but not SAT, whereas female VAT and SAT underwent hyperplastic growth. Using adipocyte progenitor transplant studies, they also demonstrated that the high-fat diet-induced differential hyperplastic growth was due to estrogen [87]. The depot-specific differences in adipogenesis were due to the adipocyte niche in which the progenitors resided, rather than cell-intrinsic mechanisms, suggesting that adipocyte progenitors are plastic cells that respond to and alter their behaviour depending on cues in their immediate environment [87]. This finding is consistent with human data showing that SAT-derived adipocyte progenitor differentiation was not different between the sexes, although females had a significantly higher proportion of early differentiated adipocytes in SAT [84]. Thus, sex- and depot-specific microenvironments coordinate adipocyte progenitor activation and ultimately adipose tissue expandability and distribution.

\section{iv. Developmental origin of WAT}

In humans, WAT develops during the second trimester of gestation [85]. Adipocytes develop from adipocyte precursors that themselves originate from mesenchymal stem cells. Although the processes controlling adipocyte precursor differentiation into mature adipocytes have been well studied, little is known about the embryonic origin of adipocytes. Lineage-tracing studies to date provide evidence that the embryonic origin of adipocytes is the mesoderm and the neural crest $[88,89]$. 
Using the 'AdipoChaser' mouse model, it has recently become possible to track adipocyte during development and to elucidate the timing of tissue formation in SAT and VAT [82]. Wang et al. [82] found that adipocytes in the gonadal fat depot differentiate postnatally and continue to develop until sexual maturation. In contrast, adipocytes in subcutaneous depots commit and differentiate during embryogenesis, between embryonic days E14-E18, and that the number of adipocytes formed in this depot remained stable thereafter. This was the case in both sexes. This observations points towards distinct developmental timings of SAT and VAT formation in vivo.

\section{Expert commentary}

i. From association to causation: implicating causal biological mechanisms in obesity

The plethora of work in obesity research is now expansive, and covers an array of approaches including genetic, epigenetic, epidemiologic, and in-vitro models. in many ways, however, we are only beginning to uncover the genetic underpinnings and causal mechanisms of obesity. GWAS findings, while capable of highlighting regions of the genome harbouring potential causal mechanisms, are extremely challenging to unravel. The vast majority of the implicated SNPs are non-coding [90,91], are correlated to tens or hundreds of other SNPs across a locus (through linkage disequilibrium (LD)), and may lie in the proximity of tens or even hundreds of genes (or even in a gene desert). It is rare that the implicated SNP itself is the causal SNP (though some examples of this phenomenon do exist [92]). Instead, the SNP is most likely tagging (i.e., in LD with) the causal variant.

As a result of GWAS locus complexity, the number of concrete connections between genetic changes and phenotypic outcomes are few. Thus, a fundamental question now faces the obesity genetics field (and indeed, the fields of many such common diseases): how do we bring observations of association to biological meaningful understanding?

\section{ii. A known causal mechanism: the FTO locus and regulation of IRX 3 and IRX5}

Though the number of known causal mechanisms is limited, a recent effort to fine-map the original discovery of the FTO locus through GWAS of BMI has shed light on one potential mechanism underpinning obesity. To identify the obesity-relevant mechanism in the FTO locus, Claussnitzer and collaborators [93] first used genetic data and bioinformatic methods to perform an in-depth analysis of the FTO locus and the genomic region surrounding it. Typically 
in genetic association analyses, a locus is defined by the span of linkage disequilibrium (i.e., correlations of SNPs) across a region. These windows are typically small, and cover $\sim 1 \mathrm{Mb}$ footprints in the genome. To better understand the potential mechanism at FTO, Claussnitzer et al. also considered Hi-C data, which measures much longer-range interactions of chromatin; regions that interact frequently are called topologically associating domains (TADs), and are thought to be key for gene regulation [94,95]. The group found that, while no potentially causal gene resided in the LD-window defined by the FTO SNP, the implicated locus resided in a TAD, discovered in human myofibroblast, that included the IRX 3 and $I R X_{5}$ genes [93].

The group then performed a series of experiments in preadipocytes derived from carriers and non-carriers of the $F T O$ risk allele, and found that perturbation of $I_{R} X_{3}$ and $I R X_{5}$ expression levels also disturbed energy balance. Overexpression of $I_{R} X_{5}$ in preadipocytes from non-risk allele carriers, for example, reduced thermogenesis to the same level as that observed in risk-allele carriers [93]; restoration of normal $I_{R} X_{5}$ expression in risk allele carriers restored normal thermogenesis. Further, the group was able to use CRISPR/Cas technology to edit the alleles of rs1421085 in preadipocytes and determine that rs1421085 is most likely the causal SNP at the locus; editing the $\mathrm{T}$ allele of $\mathrm{rs}_{1421085}$ to the $\mathrm{C}$ allele doubled $I_{R} X_{3}$ and $I_{R} X_{5}$ expression, revealing an expression profile similar to that of risk allele carriers, while editing the $\mathrm{C}$ to $\mathrm{T}$ restored normal function, but only in the presence of $A_{R I D} B$. Thus, the group inferred that carrying the rs1421085 SNP can disrupt the binding of repressor $A R I D_{5} B$, thereby increasing the overall expression of $I R X_{3}$ and $I R X_{5}$ in early adipocyte differentiation. The result of this perturbance is decreased thermogenesis, increased lipid storage and thus, an increase in body fat [93].

\section{Five-year view: towards discovery of additional causal mechanisms}

Beyond providing a compelling example of a causal mechanism in obesity, Claussnitzer and collaborators also demonstrate several key methodological advances that will likely be crucial to understanding additional obesity biology. The unraveling of the the biological mechanisms harbored in the FTO locus provides a compelling example that disease biology can be uncovered from genomic regions identified through GWAS, as well as a strategy for how to dissect these complex loci down to disease-relevant pathways. Indeed, this has already been demonstrated in other complex traits as well [92,96-99]. Yet, the many loci discovered through genome-wide scans of BMI [8], WHRadjBMI [9], and related metabolic traits [39,41,100,101] have yet to be 
unraveled in a similar manner; these loci likely flag other regions of the genome containing important regulatory pathways for obesity and adipose distribution. Second, Claussnitzer et al. demonstrate that, to bring these loci forward to biological translation and even potential clinical impact, we must improve our ability to integrate many data types together. To reveal the FTO-IRX $3-I R X_{5}-A R I D 5 B$ pathway, the group exploited the integration of several data types including GWAS data, Hi-C data, primary human cells, mouse models, and genome editing technology [93]. In essence, their effort reflects a path forward to translating GWAS signals to human biology.

While GWAS represent an efficient first step in unraveling human disease biology, it is key to note that such studies also suffer from a number of biases, which will propagate in downstream analysis and interpretation. First, a small number of GWAS efforts have focused explicitly on inclusion of multi-ancestry samples [37,102-104], and these studies have helped in fine-mapping known loci. However, the vast majority of samples collected and that have undergone GWAS are of European ancestry [105,106]; sample sizes still massively eclipse the most recent efforts in non-European studies [37,103]. This bias implies that samples studied through GWAS do not provide a global representation of common disease. In turn, development of precision medicines is shown to be biased towards disease in European-descent individuals [107]. Additionally, the lack of diverse ancestral populations in genetic studies has limited our ability to fine-map known loci. Inclusion of multi-ancestry samples in future studies will aid in our ability to fine-map these complex regions with greater precision [108-110]. GWAS studies have also been primarily focused on analysis of the autosomal chromosomes, leaving the sex chromosomes incompletely analyzed (e.g., in meta-analyses where only a fraction of the contributing samples have sex chromosome data available) or unanalyzed entirely. Given the number of sexually dimorphic loci revealed by analysis of WHRadjBMI, and the potential role of sex-specific hormone regulation in adiposity-related traits, thorough analysis of the sex chromosomes and sex-specific analyses (considering not only $\mathrm{XX}$ and $\mathrm{XY}$ individuals, but also those with other sex chromosome configurations) will likely be helpful in further unraveling adipose-related biology. Lastly, GWAS have been historically biased towards individuals measured at a single timepoint. The emergence of large-scale biobanking [111,112], and the longitudinal phenotyping often available with biobanked samples, will further enhance future genome-wide scans.

To proceed beyond GWAS findings and further unravel biology, we will need to begin 
considering more than just genetic variation, and consider the genome in more than just linear space (as is done in GWAS). For example, a number of techniques, such as $3 \mathrm{C}, 4 \mathrm{C}$, and $\mathrm{Hi}-\mathrm{C}$, can capture genome conformation and long-range interactions of distant regions of the genome occurring in three-dimensional space [94,95,113,114]. A number of other data types can also be leveraged to create a more complete picture of disease biology. RNA sequencing allows for gene expression measurements not only in blood but across a number of tissues [115,116]. Histology data now allows us to analyze cellular phenotypes. Additional imaging, such as that from MRI and CT scans, will allow us to quantify amounts of adipose tissue in specific depots of the body [117], rather than analyzing traits such as BMI or WHRadjBMI, which provide broader snapshots of overall fat distribution. Animal models will allow us to test the phenotypic effects of gene manipulations. Still-developing DNA editing tools such as CRISPR/Cas [118] will allow us to observe the phenotypic effects of specific genetic changes in cell lines derived from humans. In short, understanding the biology of obesity and obesity-related traits will likely require the integration of many data types drawn from many models to elucidate the potentially highly-complex pathways linking genetic changes or other biological perturbations to phenotypic outcome. When we can paint a more complete picture of the human body, and of the web of phenotypes and array of biological features that influence metabolic disease, we will be able to unravel complex biology, elucidate causal mechanisms, and identify drug targets. 


\section{Acknowledgements}

C.M.L. is supported by the Li Ka Shing Foundation and by the NIHR Biomedical Research

Centre, Oxford. S.L.P. is supported by the Li Ka Shing Foundation. S.L. is supported by an MRC Studentship. 


\section{Figures}

Figure 1 | Obesity and obesity-related phenotypes in humans. To study obesity and obesity-related outcomes, researchers select from a number of phenotypes to study. Anthropometric measures, such as body mass index (in kilograms/meter ${ }^{2}$ ) is one such measure, and has been used for genome-wide association studies numbering into the hundreds of thousands of samples. Waist-to-hip ratio (unadjusted or adjusted for BMI) and waist circumference are other commonly-studied body shape phenotypes. While genome-wide association studies of these anthropometric traits have yielded insights to disease biology, these phenotypes are proxies for adipose tissue residing in particular depots. Sulbcutaneous adipose tissue (SAT) collects in skin (dermal SAT) just beneath the skin (superficial SAT), in a second layer under the superficial layer (deep SAT), and in the gluteal and femoral regions. Ectopic fat is mainly found within skeletal muscle where lipids are deposited within muscle fibers (intramuscular) or within the liver (hepatic). Visceral adipose tissue (VAT) comprises the deepest layers of adipose tissue and collects around organs such as the heart (pericardial and epicardial), kidneys (renal and adrenal), liver (hepatie), gonads, vasculature (perivascular) and around the intestine in the abdominal cavity (omental, mesenteric, retroperitoneal). Organ images were created by Photoroyalty (Freepik.com). Figure adapted in part from [119]. 


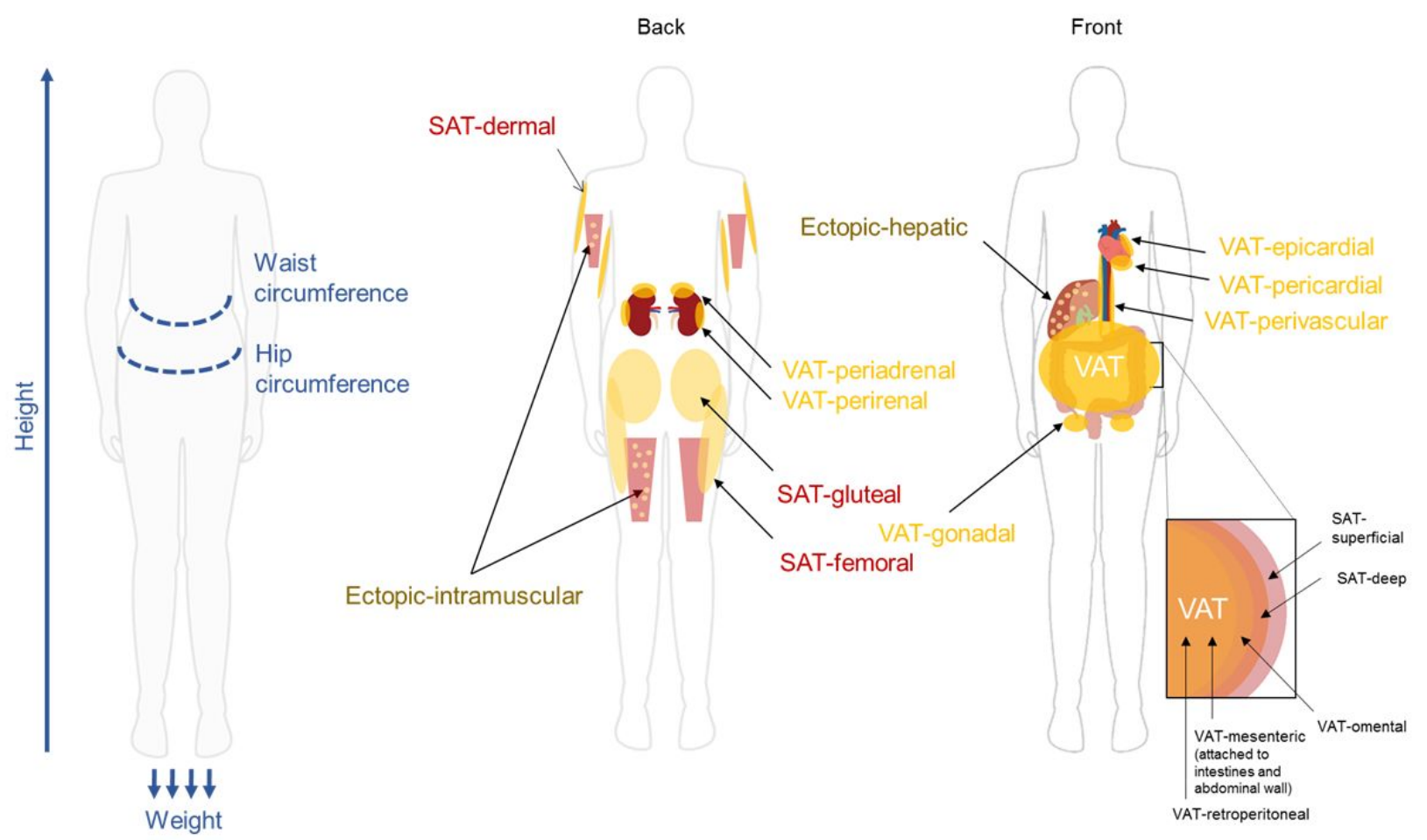


Figure 2 | The impact of a high fat diet on adipogenesis and adipocyte count and size in mice. Reports by Jeffery et al. [87] and Wang et al. [82] revealed a unique pattern of adipose tissue expansion in different depots of mice placed on a high-fat diet. Male and female mice placed on a high-fat diet for 8 weeks demonstrate sexually-dimorphic changes in diet-induced adipogenesis and increased adipocyte size. Female mice on a high fat diet showed an increase in adipocyte size (hypertrophy) in visceral adipose tissue (VAT) and an increase in total number of adipocytes (hyperplasia) in both VAT and subcutaneous adipose tissue (SAT). Male mice also displayed increased hyperplasia and hypertrophy in VAT after being placed on a high-fat diet. In contrast, male mice showed no increase in adipogenesis in SAT; instead, there was only evidence for hypertrophy in these adipocytes.

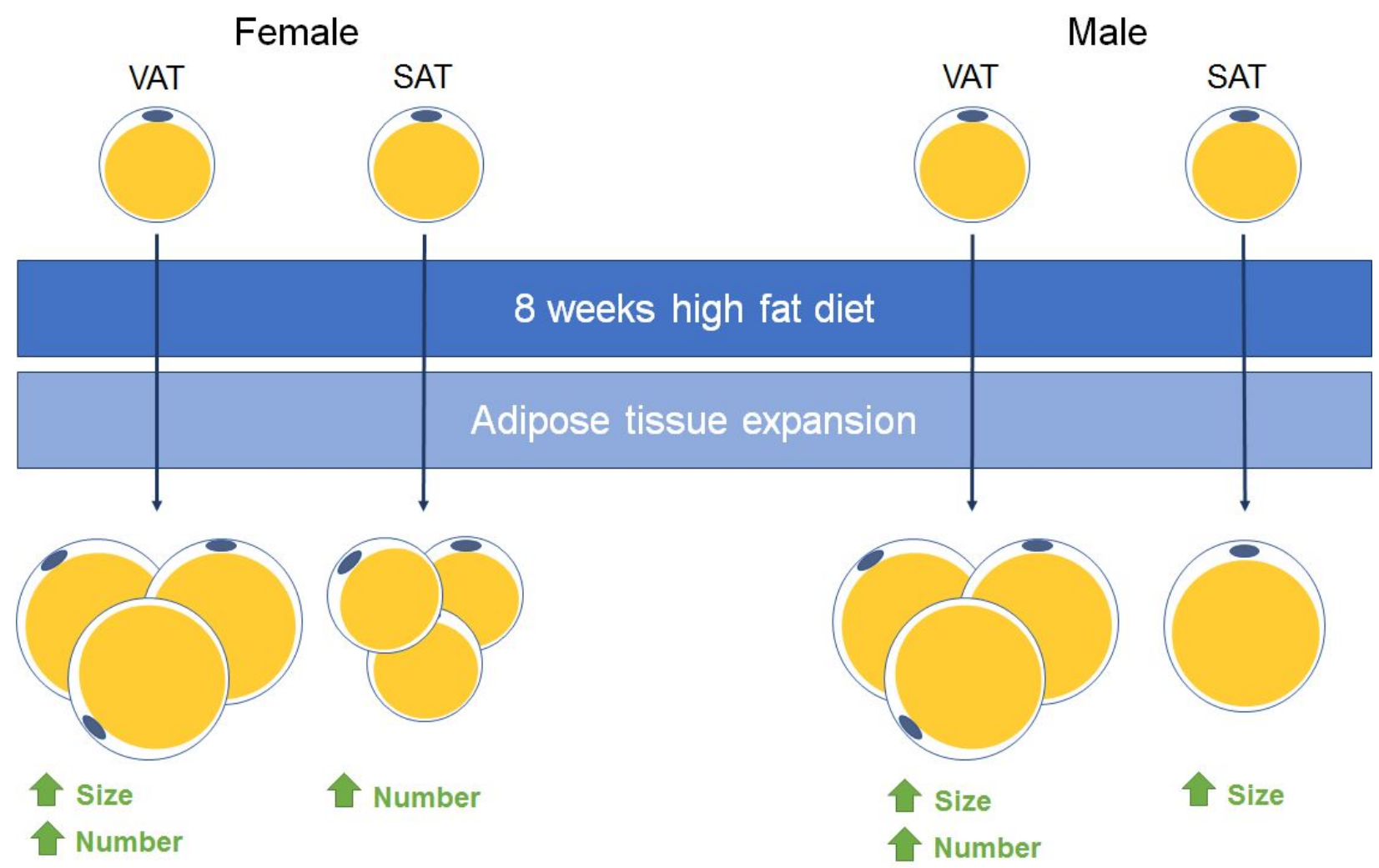




\section{References}

1. GBD 2015 Obesity Collaborators, Afshin A, Forouzanfar MH, Reitsma MB, Sur P, Estep K, et al. Health Effects of Overweight and Obesity in 195 Countries over 25 Years. N Engl J

Med. Massachusetts Medical Society; 2017;377: 13-27.

2. State of Obesity: Better Policies for a Healthier America [Internet]. Trust for America's Health; 2017. Available: https://stateofobesity.org/

3. Ng M, Fleming T, Robinson M, Thomson B, Graetz N, Margono C, et al. Global, regional, and national prevalence of overweight and obesity in children and adults during 1980-2013: a systematic analysis for the Global Burden of Disease Study 2013. Lancet. 2014;384: 766-781.

4. Prentice AM. The emerging epidemic of obesity in developing countries. Int J Epidemiol. 2006;35: 93-99.

5. Ferrara A. Increasing prevalence of gestational diabetes mellitus: a public health perspective. Diabetes Care. 2007;30 Suppl 2: S141-6.

6. Lassale C, Tzoulaki I, Moons KGM, Sweeting M, Boer J, Johnson L, et al. Separate and combined associations of obesity and metabolic health with coronary heart disease: a pan-European case-cohort analysis. Eur Heart J. 2017; doi:10.1093/eurheartj/ehx448

7. US Preventive Services Task Force, Grossman DC, Bibbins-Domingo K, Curry SJ, Barry MJ, Davidson KW, et al. Screening for Obesity in Children and Adolescents: US Preventive Services Task Force Recommendation Statement. JAMA. 2017;317: 2417-2426.

8. Locke AE, Kahali B, Berndt SI, Justice AE, Pers TH, Day FR, et al. Genetic studies of body mass index yield new insights for obesity biology. Nature. Nature Publishing Group, a division of Macmillan Publishers Limited. All Rights Reserved.; 2015;518: 197-206.

9. Shungin D, Winkler TW, Croteau-Chonka DC, Ferreira T, Locke AE, Mägi R, et al. New genetic loci link adipose and insulin biology to body fat distribution. Nature. 2015;518: 187-196.

10. Fox CS, Liu Y, White CC, Feitosa M, Smith AV, Heard-Costa N, et al. Genome-wide association for abdominal subcutaneous and visceral adipose reveals a novel locus for visceral fat in women. PLoS Genet. 2012;8: e1002695.

11. Randall JC, Winkler TW, Kutalik Z, Berndt SI, Jackson AU, Monda KL, et al. Sex-stratified genome-wide association studies including 270,000 individuals show sexual dimorphism in genetic loci for anthropometric traits. PLoS Genet. 2013;9: e1003500.

12. Aschard H, Vilhjálmsson BJ, Joshi AD, Price AL, Kraft P. Adjusting for heritable covariates can bias effect estimates in genome-wide association studies. Am J Hum Genet. 2015;96: 329-339.

13. Cole SR, Platt RW, Schisterman EF, Chu H, Westreich D, Richardson D, et al. Illustrating 
bias due to conditioning on a collider. Int J Epidemiol. 2010;39: 417-420.

14. Adams SA, Matthews CE, Ebbeling CB, Moore CG, Cunningham JE, Fulton J, et al. The effect of social desirability and social approval on self-reports of physical activity. Am J Epidemiol. 2005;161: 389-398.

15. Bell CG, Walley AJ, Froguel P. The genetics of human obesity. Nat Rev Genet. 2005;6: 221-234.

16. Farooqi S, O’Rahilly S. Genetics of obesity in humans. Endocr Rev. 2006;27: 710-718.

17. Farooqi IS, Yeo GS, Keogh JM, Aminian S, Jebb SA, Butler G, et al. Dominant and recessive inheritance of morbid obesity associated with melanocortin 4 receptor deficiency. $\mathrm{J}$ Clin Invest. 2000;106: 271-279.

18. Yeo GS, Farooqi IS, Aminian S, Halsall DJ, Stanhope RG, O’Rahilly S. A frameshift mutation in $\mathrm{MC} 4 \mathrm{R}$ associated with dominantly inherited human obesity. Nat Genet. 1998;20: 111-112.

19. Farooqi IS, Keogh JM, Yeo GSH, Lank EJ, Cheetham T, O'Rahilly S. Clinical spectrum of obesity and mutations in the melanocortin 4 receptor gene. N Engl J Med. 2003;348: 1085-1095.

20. Forsythe E, Beales PL. Bardet-Biedl syndrome. Eur J Hum Genet. Nature Publishing Group; 2012;21: 8-13.

21. Herrera BM, Lindgren CM. The genetics of obesity. Curr Diab Rep. 2010;10: 498-505.

22. Tenesa A, Haley CS. The heritability of human disease: estimation, uses and abuses. Nat Rev Genet. 2013;14: 139-149.

23. Manolio TA, Collins FS, Cox NJ, Goldstein DB, Hindorff LA, Hunter DJ, et al. Finding the missing heritability of complex diseases. Nature. 2009;461: 747-753.

24. Stunkard AJ, Foch TT, Hrubec Z. A twin study of human obesity. JAMA. 1986;256: 51-54.

25. Wood AR, Esko T, Yang J, Vedantam S, Pers TH, Gustafsson S, et al. Defining the role of common variation in the genomic and biological architecture of adult human height. Nat Genet. 2014;46: 1173-1186.

26. Hemani G, Yang J, Vinkhuyzen A, Powell JE, Willemsen G, Hottenga J-J, et al. Inference of the genetic architecture underlying BMI and height with the use of 20,240 sibling pairs. Am J Hum Genet. The American Society of Human Genetics; 2013;93: 865-875.

27. Zaitlen N, Pasaniuc B, Sankararaman S, Bhatia G, Zhang J, Gusev A, et al. Leveraging population admixture to characterize the heritability of complex traits. Nat Genet. 2014;46: 1356-1362.

28. Pulit SL, Karaderi T, Lindgren CM. Sexual dimorphisms in genetic loci linked to body fat distribution. Biosci Rep. 2017;37. doi:10.1042/BSR20160184 
29. Elks CE, den Hoed M, Zhao JH, Sharp SJ, Wareham NJ, Loos RJF, et al. Variability in the heritability of body mass index: a systematic review and meta-regression. Front Endocrinol . 2012;3: 29.

30. van Dongen J, Willemsen G, Chen W-M, de Geus EJC, Boomsma DI. Heritability of metabolic syndrome traits in a large population-based sample. J Lipid Res. 2013;54: 2914-2923.

31. Hirschhorn JN, Daly MJ. Genome-wide association studies for common diseases and complex traits. Nat Rev Genet. 2005;6: 95-108.

32. McCarthy MI, Abecasis GR, Cardon LR, Goldstein DB, Little J, Ioannidis JPA, et al. Genome-wide association studies for complex traits: consensus, uncertainty and challenges. Nat Rev Genet. 2008;9: 356-369.

33. Welter D, MacArthur J, Morales J, Burdett T, Hall P, Junkins H, et al. The NHGRI GWAS Catalog, a curated resource of SNP-trait associations. Nucleic Acids Res. 2014;42: D1001-6.

34. Frayling TM, Timpson NJ, Weedon MN, Zeggini E, Freathy RM, Lindgren CM, et al. A common variant in the FTO gene is associated with body mass index and predisposes to childhood and adult obesity. Science. 2007;316: 889-894.

35. Loos RJF, Lindgren CM, Li S, Wheeler E, Zhao JH, Prokopenko I, et al. Common variants near MC4R are associated with fat mass, weight and risk of obesity. Nat Genet. 2008;40: $768-775$.

36. Willer CJ, Speliotes EK, Loos RJF, Li S, Lindgren CM, Heid IM, et al. Six new loci associated with body mass index highlight a neuronal influence on body weight regulation. Nat Genet. 2009;41: 25-34.

37. Chu AY, Deng X, Fisher VA, Drong A, Zhang Y, Feitosa MF, et al. Multiethnic genome-wide meta-analysis of ectopic fat depots identifies loci associated with adipocyte development and differentiation. Nat Genet. 2017;49: 125-130.

38. Pers TH, Karjalainen JM, Chan Y, Westra H-J, Wood AR, Yang J, et al. Biological interpretation of genome-wide association studies using predicted gene functions. Nat Commun. 2015;6: 5890 .

39. Fuchsberger C, Flannick J, Teslovich TM, Mahajan A, Agarwala V, Gaulton KJ, et al. The genetic architecture of type 2 diabetes. Nature. 2016;536: 41-47.

40. Teslovich TM, Musunuru K, Smith AV, Edmondson AC, Stylianou IM, Koseki M, et al. Biological, clinical and population relevance of 95 loci for blood lipids. Nature. 2010;466: 707-713.

41. Willer CJ, Schmidt EM, Sengupta S, Peloso GM, Gustafsson S, Kanoni S, et al. Discovery and refinement of loci associated with lipid levels. Nat Genet. 2013;45: 1274-1283.

42. Wahl S, Drong A, Lehne B, Loh M, Scott WR, Kunze S, et al. Epigenome-wide association study of body mass index, and the adverse outcomes of adiposity. Nature. 2017;541: 81-86. 
43. Karaderi T, Drong AW, Lindgren CM. Insights into the Genetic Susceptibility to Type 2 Diabetes from Genome-Wide Association Studies of Obesity-Related Traits. Curr Diab Rep. 2015;15: 83 .

44. Gaulton KJ, Ferreira T, Lee Y, Raimondo A, Mägi R, Reschen ME, et al. Genetic fine mapping and genomic annotation defines causal mechanisms at type 2 diabetes susceptibility loci. Nat Genet. 2015;47: 1415-1425.

45. Dahlman I, Rydén M, Brodin D, Grallert H, Strawbridge RJ, Arner P. Numerous Genes in Loci Associated With Body Fat Distribution Are Linked to Adipose Function. Diabetes. 2016;65: 433-437.

46. Claussnitzer M, Dankel SN, Klocke B, Grallert H, Glunk V, Berulava T, et al. Leveraging cross-species transcription factor binding site patterns: from diabetes risk loci to disease mechanisms. Cell. 2014;156: 343-358.

47. Pischon T, Boeing H, Hoffmann K, Bergmann M, Schulze MB, Overvad K, et al. General and abdominal adiposity and risk of death in Europe. N Engl J Med. 2008;359: 2105-2120.

48. Wormser D, Kaptoge S, Di Angelantonio E, Wood AM, Pennells L, Thompson A, et al. Emerging Risk Factors Collaboration: Separate and combined associations of body-mass index and abdominal adiposity with cardiovascular disease: collaborative analysis of 58 prospective studies. Lancet. 2011;377: 1085-1095.

49. Coutinho T, Goel K, Corrêa de Sá D, Kragelund C, Kanaya AM, Zeller M, et al. Central Obesity and Survival in Subjects With Coronary Artery Disease: A Systematic Review of the Literature and Collaborative Analysis With Individual Subject Data. J Am Coll Cardiol. 2011;57: 1877-1886.

50. Phillips LK, Prins JB. The link between abdominal obesity and the metabolic syndrome. Curr Hypertens Rep. 2008;10: 156-164.

51. Nazare J-A, Smith JD, Borel A-L, Haffner SM, Balkau B, Ross R, et al. Ethnic influences on the relations between abdominal subcutaneous and visceral adiposity, liver fat, and cardiometabolic risk profile: the International Study of Prediction of Intra-Abdominal Adiposity and Its Relationship With Cardiometabolic Risk/Intra-Abdominal Adiposity. Am J Clin Nutr. Am Soc Nutrition; 2012;96: 714-726.

52. Lee B-C, Lee J. Cellular and molecular players in adipose tissue inflammation in the development of obesity-induced insulin resistance. Biochim Biophys Acta. 2014;1842: 446-462.

53. Misra A, Garg A, Abate N, Peshock RM, Stray-Gundersen J, Grundy SM. Relationship of anterior and posterior subcutaneous abdominal fat to insulin sensitivity in nondiabetic men. Obes Res. 1997;5: 93-99.

54. Snijder MB, Dekker JM, Visser M, Bouter LM, Stehouwer CDA, Kostense PJ, et al. Associations of hip and thigh circumferences independent of waist circumference with the incidence of type 2 diabetes: the Hoorn Study. Am J Clin Nutr. 2003;77: 1192-1197. 
55. Tran TT, Yamamoto Y, Gesta S, Kahn CR. Beneficial effects of subcutaneous fat transplantation on metabolism. Cell Metab. 2008;7: 410-420.

56. Appleton SL, Seaborn CJ, Visvanathan R, Hill CL, Gill TK, Taylor AW, et al. Diabetes and Cardiovascular Disease Outcomes in the Metabolically Healthy Obese Phenotype. Diabetes Care. American Diabetes Association; 2013;36: 2388-2394.

57. Heitmann BL, Lissner L. Hip Hip Hurrah! Hip size inversely related to heart disease and total mortality. Obes Rev. 2011;12: 478-481.

58. Manolopoulos KN, Karpe F, Frayn KN. Gluteofemoral body fat as a determinant of metabolic health. Int J Obes . 2010;34: 949-959.

59. Stefan N, Fritsche A, Schick F, Häring H-U. Phenotypes of prediabetes and stratification of cardiometabolic risk. Lancet Diabetes Endocrinol. 2016;4: 789-798.

6o. Stefan N, Schick F, Häring H-U. Causes, Characteristics, and Consequences of Metabolically Unhealthy Normal Weight in Humans. Cell Metab. 2017;26: 292-300.

61. Wajchenberg BL, Giannella-Neto D, da Silva ME, Santos RF. Depot-specific hormonal characteristics of subcutaneous and visceral adipose tissue and their relation to the metabolic syndrome. Horm Metab Res. 2002;34: 616-621.

62. Gesta S, Blüher M, Yamamoto Y, Norris AW, Berndt J, Kralisch S, et al. Evidence for a role of developmental genes in the origin of obesity and body fat distribution. Proc Natl Acad Sci U S A. 2006;103: 6676-6681.

63. Yamamoto Y, Gesta S, Lee KY, Tran TT, Saadatirad P, Kahn CR. Adipose depots possess unique developmental gene signatures. Obesity . 2010;18: 872-878.

64. Cantile M, Procino A, D’Armiento M, Cindolo L, Cillo C. HOX gene network is involved in the transcriptional regulation of in vivo human adipogenesis. J Cell Physiol. 2003;194: $225-236$.

65. Cohen M, Syme C, Deforest M, Wells G, Detzler G, Cheng H-L, et al. Ectopic fat in youth: the contribution of hepatic and pancreatic fat to metabolic disturbances. Obesity . 2014;22: 1280-1286.

66. Grove KL, Fried SK, Greenberg AS, Xiao XQ, Clegg DJ. A microarray analysis of sexual dimorphism of adipose tissues in high-fat-diet-induced obese mice. Int J Obes . 2010;34: 989-1000.

67. Karastergiou K, Fried SK, Xie H, Lee M-J, Divoux A, Rosencrantz MA, et al. Distinct developmental signatures of human abdominal and gluteal subcutaneous adipose tissue depots. J Clin Endocrinol Metab. 2013;98: 362-371.

68. Chau Y-Y, Bandiera R, Serrels A, Martínez-Estrada OM, Qing W, Lee M, et al. Visceral and subcutaneous fat have different origins and evidence supports a mesothelial source. Nat Cell Biol. 2014;16: 367-375.

69. Krueger KC, Costa MJ, Du H, Feldman BJ. Characterization of Cre recombinase activity for 
in vivo targeting of adipocyte precursor cells. Stem Cell Reports. 2014;3: 1147-1158.

70. Sanchez-Gurmaches J, Guertin DA. Adipocytes arise from multiple lineages that are heterogeneously and dynamically distributed. Nat Commun. 2014;5: 4099.

71. Palmer BF, Clegg DJ. The sexual dimorphism of obesity. Mol Cell Endocrinol. 2015;402: 113-119.

72. Gesta S, Tseng Y-H, Kahn CR. Developmental origin of fat: tracking obesity to its source. Cell. 2007;131: 242-256.

73. Berry R, Jeffery E, Rodeheffer MS. Weighing in on adipocyte precursors. Cell Metab. 2014;19: 8-20.

74. Weyer C, Foley JE, Bogardus C, Tataranni PA, Pratley RE. Enlarged subcutaneous abdominal adipocyte size, but not obesity itself, predicts type II diabetes independent of insulin resistance. Diabetologia. 2000;43: 1498-1506.

75. Skurk T, Alberti-Huber C, Herder C, Hauner H. Relationship between adipocyte size and adipokine expression and secretion. J Clin Endocrinol Metab. 2007;92: 1023-1033.

76. Fang L, Guo F, Zhou L, Stahl R, Grams J. The cell size and distribution of adipocytes from subcutaneous and visceral fat is associated with type 2 diabetes mellitus in humans.

Adipocyte. 2015;4: 273-279.

77. Spalding KL, Arner E, Westermark PO, Bernard S, Buchholz BA, Bergmann O, et al. Dynamics of fat cell turnover in humans. Nature. 2008;453: 783-787.

78. Choe SS, Huh JY, Hwang IJ, Kim JI, Kim JB. Adipose Tissue Remodeling: Its Role in Energy Metabolism and Metabolic Disorders. Front Endocrinol . 2016;7: 30.

79. Wernstedt Asterholm I, Tao C, Morley TS, Wang QA, Delgado-Lopez F, Wang ZV, et al. Adipocyte inflammation is essential for healthy adipose tissue expansion and remodeling. Cell Metab. 2014;20: 103-118.

80. Jeffery E, Church CD, Holtrup B, Colman L, Rodeheffer MS. Rapid depot-specific activation of adipocyte precursor cells at the onset of obesity. Nat Cell Biol. 2015;17: 376-385.

81. Arner E, Westermark PO, Spalding KL, Britton T, Rydén M, Frisén J, et al. Adipocyte turnover: relevance to human adipose tissue morphology. Diabetes. 2010;59: 105-109.

82. Wang QA, Tao C, Gupta RK, Scherer PE. Tracking adipogenesis during white adipose tissue development, expansion and regeneration. Nat Med. 2013;19: 1338-1344.

83. Kim SM, Lun M, Wang M, Senyo SE, Guillermier C, Patwari P, et al. Loss of white adipose hyperplastic potential is associated with enhanced susceptibility to insulin resistance. Cell Metab. 2014;20: 1049-1058.

84. Tchoukalova YD, Koutsari C, Votruba SB, Tchkonia T, Giorgadze N, Thomou T, et al. Sexand depot-dependent differences in adipogenesis in normal-weight humans. Obesity . 
2010;18: 1875-1880.

85. Pellegrinelli V, Carobbio S, Vidal-Puig A. Adipose tissue plasticity: how fat depots respond differently to pathophysiological cues. Diabetologia. 2016;59: 1075-1088.

86. Harms M, Seale P. Brown and beige fat: development, function and therapeutic potential. Nat Med. 2013;19: 1252-1263.

87. Jeffery E, Wing A, Holtrup B, Sebo Z, Kaplan JL, Saavedra-Peña R, et al. The Adipose Tissue Microenvironment Regulates Depot-Specific Adipogenesis in Obesity. Cell Metab. 2016;24: 142-150.

88. Billon N, Iannarelli P, Monteiro MC, Glavieux-Pardanaud C, Richardson WD, Kessaris N, et al. The generation of adipocytes by the neural crest. Development. 2007;134: 2283-2292.

89. Sanchez-Gurmaches J, Hung C-M, Guertin DA. Emerging Complexities in Adipocyte Origins and Identity. Trends Cell Biol. 2016;26: 313-326.

90. Zhang F, Lupski JR. Non-coding genetic variants in human disease. Hum Mol Genet. 2015;24: R102-10.

91. Manolio TA. Bringing genome-wide association findings into clinical use. Nat Rev Genet. Nature Publishing Group; 2013;14: 549-558.

92. Gregory AP, Dendrou CA, Attfield KE, Haghikia A, Xifara DK, Butter F, et al. TNF receptor 1 genetic risk mirrors outcome of anti-TNF therapy in multiple sclerosis. Nature. 2012;488: 508-511.

93. Claussnitzer M, Dankel SN, Kim K-H, Quon G, Meuleman W, Haugen C, et al. FTO Obesity Variant Circuitry and Adipocyte Browning in Humans. N Engl J Med. 2015;373: 895-907.

94. Dixon JR, Selvaraj S, Yue F, Kim A, Li Y, Shen Y, et al. Topological domains in mammalian genomes identified by analysis of chromatin interactions. Nature. 2012;485: 376-380.

95. Grubert F, Zaugg JB, Kasowski M, Ursu O, Spacek DV, Martin AR, et al. Genetic Control of Chromatin States in Humans Involves Local and Distal Chromosomal Interactions. Cell. 2015;162: 1051-1065.

96. Abifadel M, Varret M, Rabès J-P, Allard D, Ouguerram K, Devillers M, et al. Mutations in PCSK9 cause autosomal dominant hypercholesterolemia. Nat Genet. 2003;34: 154-156.

97. Kathiresan S, Voight BF, Purcell S, Musunuru K, Ardissino D, Mannucci PM, et al. Genome-wide association of early-onset myocardial infarction with single nucleotide polymorphisms and copy number variants. Nat Genet. 2009;41: 334-341.

98. Ripke S, Neale BM, Corvin A, Walters JTR, Farh K-H, Holmans P a., et al. Biological insights from 108 schizophrenia-associated genetic loci. Nature. 2014;511: 421-427.

99. Sekar A, Bialas AR, de Rivera H, Davis A, Hammond TR, Kamitaki N, et al. Schizophrenia risk from complex variation of complement component 4. Nature. Nature Publishing 
Group; 2016;530: 177-183.

100. Ehret GB, Ferreira T, Chasman DI, Jackson AU, Schmidt EM, Johnson T, et al. The genetics of blood pressure regulation and its target organs from association studies in 342,415 individuals. Nat Genet. 2016;48: 1171-1184.

101. Lauenborg J, Grarup N, Damm P, Borch-Johnsen K, Jørgensen T, Pedersen O, et al. Common type 2 diabetes risk gene variants associate with gestational diabetes. J Clin Endocrinol Metab. 2009;94: 145-150.

102. Monda KL, Chen GK, Taylor KC, Palmer C, Edwards TL, Lange LA, et al. A meta-analysis identifies new loci associated with body mass index in individuals of African ancestry. Nat Genet. 2013;45: 690-696.

103. Ng MCY, Graff M, Lu Y, Justice AE, Mudgal P, Liu C-T, et al. Discovery and fine-mapping of adiposity loci using high density imputation of genome-wide association studies in individuals of African ancestry: African ancestry anthropometry genetics consortium. PLoS Genet. 2017;13: e1006719.

104. Liu C-T, Monda KL, Taylor KC, Lange L, Demerath EW, Palmas W, et al. Genome-wide association of body fat distribution in African ancestry populations suggests new loci. PLoS Genet. 2013;9: e1003681.

105. Rosenberg NA, Huang L, Jewett EM, Szpiech ZA, Jankovic I, Boehnke M. Genome-wide association studies in diverse populations. Nat Rev Genet. 2010;11: 356-366.

106. Pulit SL, Voight BF, de Bakker PIW. Multiethnic genetic association studies improve power for locus discovery. PLoS One. 2010;5: e12600.

107. Petrovski S, Goldstein DB. Unequal representation of genetic variation across ancestry groups creates healthcare inequality in the application of precision medicine. Genome Biol. 2016;17: 157 .

108. Zaitlen N, Paşaniuc B, Gur T, Ziv E, Halperin E. Leveraging Genetic Variability across Populations for the Identification of Causal Variants. Am J Hum Genet. 2010;86: 23-33.

109. Morris AP. Transethnic meta-analysis of genomewide association studies. Genet Epidemiol. 2011;35: 809-822.

110. van de Bunt M, Cortes A, Brown MA, Morris AP, McCarthy MI. Evaluating the Performance of Fine-Mapping Strategies at Common Variant GWAS Loci. PLoS Genet. 2015;11: e1005535.

111. Sudlow C, Gallacher J, Allen N, Beral V, Burton P, Danesh J, et al. UK biobank: an open access resource for identifying the causes of a wide range of complex diseases of middle and old age. PLoS Med. 2015;12: e1001779.

112. Siva N. UK gears up to decode 100,000 genomes from NHS patients. Lancet. $2015 ; 385$ : 103-104.

113. Nagano T, Lubling Y, Yaffe E, Wingett SW, Dean W, Tanay A, et al. Single-cell Hi-C for 
genome-wide detection of chromatin interactions that occur simultaneously in a single cell. Nat Protoc. 2015;10: 1986-2003.

114. Rao SSP, Huntley MH, Durand NC, Stamenova EK, Bochkov ID, Robinson JT, et al. A 3D map of the human genome at kilobase resolution reveals principles of chromatin looping. Cell. 2014;159: 1665-1680.

115. GTEx Consortium. Human genomics. The Genotype-Tissue Expression (GTEx) pilot analysis: multitissue gene regulation in humans. Science. 2015;348: 648-660.

116. Lonsdale J, Thomas J, Salvatore M, Phillips R, Lo E, Shad S, et al. The Genotype-Tissue Expression (GTEx) project. Nat Genet. 2013;45: 580-585.

117. Benfield LL, Fox KR, Peters DM, Blake H, Rogers I, Grant C, et al. Magnetic resonance imaging of abdominal adiposity in a large cohort of British children. Int J Obes . 2008;32: 91-99.

118. Cong L, Ran FA, Cox D, Lin S, Barretto R, Habib N, et al. Multiplex genome engineering using CRISPR/Cas systems. Science. 2013;339: 819-823.

119. Chusyd DE, Wang D, Huffman DM, Nagy TR. Relationships between Rodent White Adipose Fat Pads and Human White Adipose Fat Depots. Front Nutr. 2016;3: 10.

[1]** An expansive, up-to-date look at obesity worldwide and the global impacts of the obesity epidemic.

[8]** The largest genome-wide association studies performed to date in body mass index (BMI), identifying 97 cumulative associated loci and enrichment in neurological pathways.

[9] ** The largest genome-wide association studies performed to date in waist-to-hip ratio (WHR), identifying 49 cumulative associated loci (nearly half of them sexually dimorphic) and enrichment in adipose-related pathways.

[12] * An important look at how adjustment for covariates -- commonly applied in the study of adiposity -- can affect results and interpretations of genetic studies.

[19]* A detailed look at the role of the $M C 4 R$ gene in syndromic obesity.

[31]* A helpful and clear look at the implementation of genome-wide association studies, for those more unfamiliar with the topic.

[32]* A second detailed review covering the implementation of genome-wide association studies, interpretation, and common pitfalls.

[42] ** A large-scale study of the role of gene methylation in adiposity, including an analysis indicating that increased adiposity perturbs gene methylation (rather 
than vice versa).

[62]* A close look at the developmental genes that appear to play a role in adiposity distribution.

[77]** An analysis of adipocytes in obese and lean adults, linking how the total number of adipocytes in an individual directly relates to overall fat mass.

[93]** A demonstration of how genome-wide association study data, integrated with additional epigenomic data, animal models, and genome editing can help elucidate biological mechanisms in adiposity.

[109] * A look at how leveraging multi-ancestry populations can improve fine-mapping at common disease loci.

[110]* A second helpful fine-mapping paper, looking at various tools available in the field for identifying potentially causal variants at loci associated to common disease. 